\title{
MAGNETOENCEPHALOGRAPHY DURING SIMULATED DRIVING: A NEW PARADIGM FOR DRIVER ASSESSMENT
}

\author{
Elizabeth A. Walshe ${ }^{1}$, Flaura K. Winston ${ }^{2}$, Chelsea Ward McIntosh ${ }^{2}$, Dan Romer ${ }^{1}$, \\ Timothy Roberts ${ }^{2}$, William Gaetz ${ }^{2}$ \\ ${ }^{1}$ Annenberg Public Policy Center, University of Pennsylvania, Philadelphia, PA, USA \\ 2 The Children's Hospital of Philadelphia, PA, USA. \\ Email: walshee@email.chop.edu
}

\begin{abstract}
Summary: Increasingly, vehicles are equipped with assistive devices and advanced warning systems to mitigate driver errors, which account for $94 \%$ of motor vehicle crashes. However, these technologies require humans to appropriately respond or take over the vehicle. If we want to design effective aids, we need to better understand the neural mechanisms underlying driver error and test how the brain responds to countermeasures. For this, we need sensitive measures of brain activity during driving. This paper present a new paradigm for driver assessment, using magnetoencephalographic (MEG) recording of whole cortex neural oscillatory activity while participants undergo an ecologicallyrelevant simulated driving experience of graded complexity. A pilot experiment set out to demonstrate that expected and motor cortex responses to basic drivingrelated movements (without salient cues) could be recorded, without significant artifact. Following this, a preliminary study of adults $(n=5)$ explored if additional cognitive neural responses to increasing driving task demands can be identified. This paradigm was successfully piloted and preliminary results reveal localized brain regions of expected motor cortex activity, as well as power increases in the frontal lobe. This paradigm can be used to identify not only the neural mechanisms underlying driver errors, but also measure the impact of assistive and alert/warning technologies on these mechanisms in both typical and impaired populations of drivers.
\end{abstract}

\section{BACKGROUND}

Everyday driving is a complex behavior that requires integrated brain function across sensory, motor and cognitive systems. For example, driving relies on multisensory processing, motor control, sustained attention, cognitive control. This allows the driver to control the vehicle, manage distractions, maintain good situational awareness, and to make quick decisions and rapid responses in critical moments to avoid crashing. Yet there are limits to the neurocognitive capacities needed for safe driving, most clearly evidenced by the detrimental effects of visual, motor and cognitive distractions on driver performance (Schweizer et al., 2013; Young et al., 2004). These limited capacities may underlie many typical driver errors, which account for $94 \%$ of motor vehicle crashes (National Highway Traffic Safety Administration, 2015). Furthermore, driver errors may vary depending on the specific traits and states of the driver (Romer et al., 2014), or known neurocognitive impairments in clinical populations of drivers.

Given this, and the advancement in automotive in-vehicle technology, assistive devices and advanced warning systems have been developed to help drivers to maintain attention on the roadway, avoid collisions and going off-road, and even to prepare drivers for manual take-over 
in autonomous driving vehicles (Lerner et al., 2015; Mosquet, Andersen \& Arora, 2016). However, in order to be effective, these interventions need to be optimized: we must understand which specific brain systems or functions are failing when drives make errors, in order to inform the design of technologies so that they meet the edges of neurocognitive capacity limits, and aid (rather than hinder) responsiveness in critical moments on the road (Lees et al., 2010).

However, to date, we know very little about the neural mechanisms and integrated function underlying safe driving and driver error. A neuroergonomic approach can be utilized to provide a greater understanding of driver error and responses to new technology, either by adapting the neuroimaging technology to real-world driving (on-road), or through the use of simulated driving tasks (Lees et al., 2010). The ideal paradigm would allow us to measure reliable whole cortex activity during on-road driving that exposes drivers to situations where critical driver errors occur. Thus, the underlying mechanisms of critical on-road driving could be quantified, and the impact of in-vehicle technology interventions on neurocognitive responses could be determined. In lieu of this, combining neuroimaging tools with simulated driving scenarios offers a way to safely expose drivers to complex scenarios to examine critical driving errors and newly developed countermeasures.

A range of neuroimaging technologies can and have been used to examine driving, including: functional Magnetic Resonance Imaging (fMRI: Calhoun et al., 2002), functional Near Infrared Spectroscopy (fNIRS: Liu, Pelowski, Pang, Zhou \& Cai, 2016), electroencephalography (EEG: Zhang et al., 2015), and to a lesser extent, magnetoencephalography (MEG: Sakihara et al., 2014). Each of these technologies has strengths and limitations in terms of sensitivity for measuring brain activity, accessibility and practical implementation for driving research (see Table 1).

Table 1. Overview of applicable neuroimaging technologies

\begin{tabular}{cccccc}
\hline $\begin{array}{c}\text { Neuroimaging } \\
\text { methods }\end{array}$ & $\begin{array}{c}\text { Activity } \\
\text { measured }\end{array}$ & $\begin{array}{c}\text { Direct/ } \\
\text { indirect }\end{array}$ & $\begin{array}{c}\text { Temporal } \\
\text { resolution }\end{array}$ & $\begin{array}{c}\text { Spatial } \\
\text { Resolution }\end{array}$ & $\begin{array}{c}\text { Driver } \\
\text { Position }\end{array}$ \\
\hline fMRI & Metabolic & Indirect & $\sim 1 \mathrm{~s}$ & $\sim 1 \mathrm{~mm}$ & Supine \\
fNIRS & Metabolic & Indirect & $\sim 1 \mathrm{~s}$ & $\sim 5 \mathrm{~mm}$ & Seated \\
EEG & Electrical & Direct & $\sim 1 \mathrm{~ms}$ & $\sim 10 \mathrm{~mm}$ & Seated \\
MEG & Magnetic & Direct & $\sim 1 \mathrm{~ms}$ & $\sim 5 \mathrm{~mm}$ & Seated
\end{tabular}

MEG has many strengths as a neuroimaging tool, yet it has been less utilized in the field of driving research, particularly with driving simulation (with wheels and pedals: Sakihara et al., 2014). MEG is a direct measure of brain function, unlike other neuroimaging technology like fMRI or FNIRS which are secondary measures of brain activity inferred from metabolic changes in the brain as a result of neuron activity. Because MEG is a direct measure of neural activity, the temporal resolution is substantially higher than fMRI and FNIRS: MEG offers millisecondsensitivity, compared to a 2-3 second lag with fMRI/FNIRS recording. In addition, new methods using MRI images to localize the source of MEG waveforms make the spatial resolution drastically higher than measures such as EEG and FNIRS, and higher still than fMRI. This paper presents a new paradigm that has married MEG and novel ecologically-relevant driving 
simulation (supplemented with MRI) for a much more accurate mapping of where and when the brain effectively responds to, or fails to support, the demands of the driving task.

Using simulated driving software allows us to strategically isolate and manipulate the wellcharacterized components of driving performance (accelerating/braking and steering), while manipulating the scene to systematically build complexity and increasingly tax integrated brain functioning and capacity. The goal of this paper is to test the feasibility of using this paradigm. As a first step, we conducted a pilot test (Experiment 1) to demonstrate that this paradigm can probe well characterized motor cortical responses (in the established hand and foot areas of the sensorimotor cortex) to basic driving-related movements (without salient cues) without significant artifact. We hypothesized that we would see standard beta-band power decreases (Gaetz et al., 2010) in contralateral hand and foot areas of the motor and sensorimotor cortex related to steering hand movement and pedal foot movements. Following this, we recruited a preliminary sample of adults (Experiment 2) to begin to characterize the visual, motor and cognitive neural responses to ecologically relevant driving behaviors, in a systematic way, using an MEG-compatible driving simulator.

\section{METHODS}

Participants. An initial pilot test (Experiment 1$)$ was conducted with a healthy adult male $(\mathrm{n}=1)$. Following this, a preliminary sample of healthy male adults was recruited and scanned for Experiment $2(\mathrm{n}=5)$ : all right-handed, licensed drivers, free from neurological or psychiatric symptoms (median age: 22 years, range: $20-24$ ).

Driving Simulation. A customized MEG-compatible driving simulator package (Current Designs, Inc.) provides a steering wheel, brake, and gas pedal that are MRI- and MEG- compatible by virtue of containing no metal, no wires, and no electronics within the components placed in the imaging suite. Light is sent from an interface unit (outside the shielded room) through a long optical fiber bundle to sensors on the driving controls and is transmitted back to the interface unit, where the detected signals are converted into standard USB control signals for use with the simulation computer. The gas and brake signals are provided as analog signals for recording in lockstep time

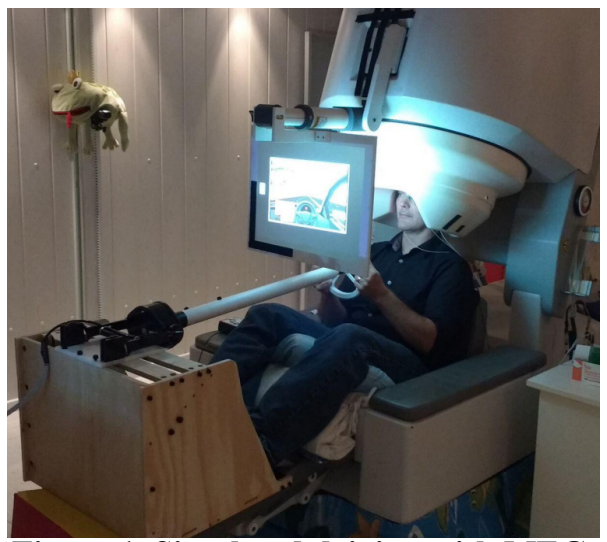

Figure 1. Simulated driving with MEG recording. series with MEG data. We developed a prototype chassis to integrate both the steering and pedal components on one base. This hardware interfaces with Diagnostic Driving Inc. software that delivers custom-built driving scenarios for this study (developed in unity3d programming environment), featuring driving tasks of graduated complexity.

For Experiment 1, on a simple straight roadway without other vehicles or pedestrians, basic motor movements of driving were performed in the absence of salient environmental cues: i) accelerating, ii) braking and iii) turning the wheel left and right. For Experiment 2, participants completed 3 blocks of basic driving scenario tasks (with repeat trials) of varying complexity: Drive1) starting and stopping at traffic light intersections on a straight roadway (speed 
management), Drive2) turning at traffic light intersections (wheel and speed management), Drive3) driving on curved road segments (requiring constant visuo-motor updating and integration for speed and steering control). Each block consisted of repeated rest period trials ( 9 seconds) and driving trials. During the rest period (control phase), the drivers were instructed to look at the rest screen and to not perform driving tasks. In-built triggers for the MEG data marked when each rest period ended and when the traffic lights changed color, which were used for defining the event/cue-related epochs for MEG analysis.

$M E G / M R I$ recording. We recorded neuromagnetic data continuously during the simulated driving tasks in a $4 \mathrm{~m} \times 5 \mathrm{~m}$ magnetically shielded room (MSR) using a 275-channel whole cortex MEG system (CTF International). Data was recorded at $1200 \mathrm{~Hz} / \mathrm{channel}$, and with a 3rd order gradiometer environmental noise reduction. Gross subject motion was identified using online head motion detection (obtained via active fiducials) so that data acquisition could be stopped and restarted if gross motion exceeded nominal thresholds $(10 \mathrm{~mm})$. After driving, a volumetric MRI was conducted using a 3T Siemens Prisma ${ }^{\mathrm{TM}}$ MR scanner which was used both for MEG source localization, and grey and white matter ROI voxel segmentation and quantification.

MEG/MRI Analysis. Following data cleaning/preprocessing (removing trials containing artifacts $>3 \mathrm{pT}$ ), the MEG data was organized into epochs $2 \mathrm{~s}$ long around the time of stimulus onset (traffic light change) or motor-response (steering, gas or brake: "active" states), for comparison to a $2 \mathrm{~s}$ epoch of baseline activity selected from 9s of rest before each trial begins ("control" state). Following previously published methods from our group, visual alpha-band, motor betaband and frontal gamma- and theta-band responses were localized using differential Synthetic Aperture Magnetometry (SAM) beamformer methods (Gaetz et al., 2010; Vrba \& Robinson, 2001; Robinson \& Vrba, 2000). This method allows for statistically significant difference images in "active" and "control" states to be compared for the specified epochs (by contrasting voxels throughout the 3-D brain source model) (Papadelis et al., 2016). For example, movementassociated areas of functional motor cortex were localized by contrasting desynchronizing oscillations in response to an "active" movement of steering, accelerating, or braking (known as event-related desynchronization), to a pre-stimulus baseline resting rhythm (typically between $15-30 \mathrm{~Hz}$ for resting beta-band). Peak increases and decreases in source power can be observed with high spatial resolution $(<0.5 \mathrm{~cm})$ at the specified frequency bands. Source waveforms from SAM peak locations were also analyzed using time-frequency wavelet methods (Tallon-Baudry, 2012) to analyze frequency, power, latency and duration changes in sensory, motor and frontal/cognitive responses. For experiment 2 , the grand average difference waves were calculated from the MEG source localization data across the group of participants $(n=5)$.

\section{RESULTS}

Head motion. Looking across the more engaging ecologically-relevant simulated driving tasks in Experiment 2 - requiring hand and foot motion for steering and the gas/brake pedals - we found that we could record neural MEG responses for each driving scenario without excessive head motion or environmental noise: Gross head motion was $\leq 1 \mathrm{~cm}$ for each case $(\mathrm{n}=5)$, range: .02 $1.0 \mathrm{~cm}$. Data acquisition did not have to be repeated due to head motion for any participants. 
Experiment 1. Comparing rest and active driving epochs, we observed beta-band event-related desynchrony (ERD) in contralateral areas of the motor cortex when the left and right hands were used to control steering, and also when the right foot was used to control the pedals. Figure 2 illustrates the accelerometer trace-defined epochs for the active and control states (left) and the differential images of the motor cortex activity (right).

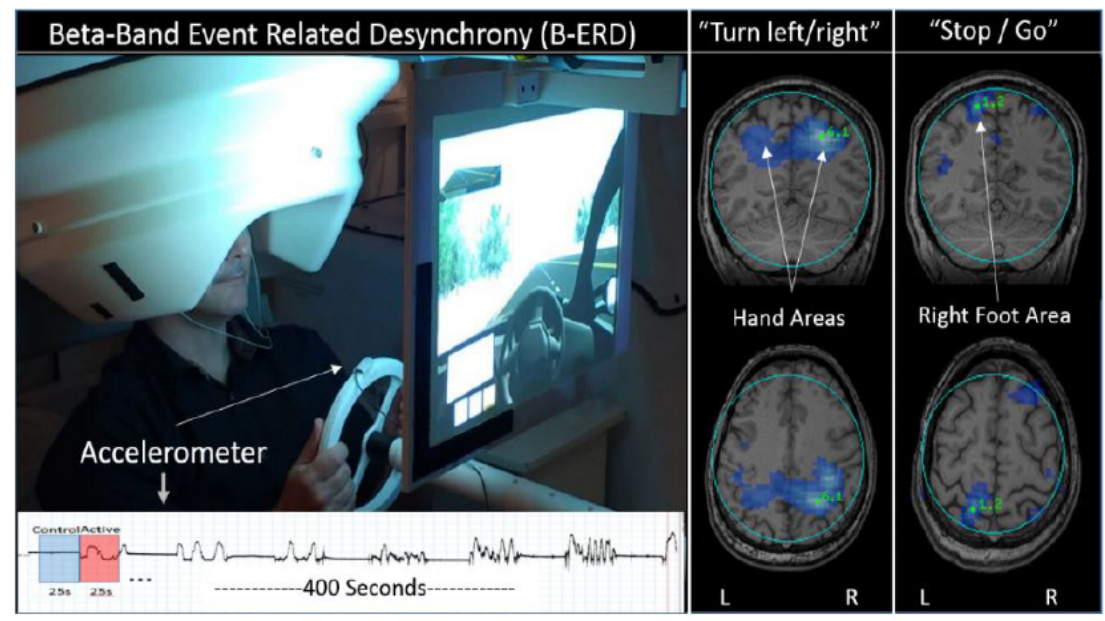

Figure 2. Simulated Driving and MEG: Expected event-related desynchrony beta-band responses associated with steer and brake events.

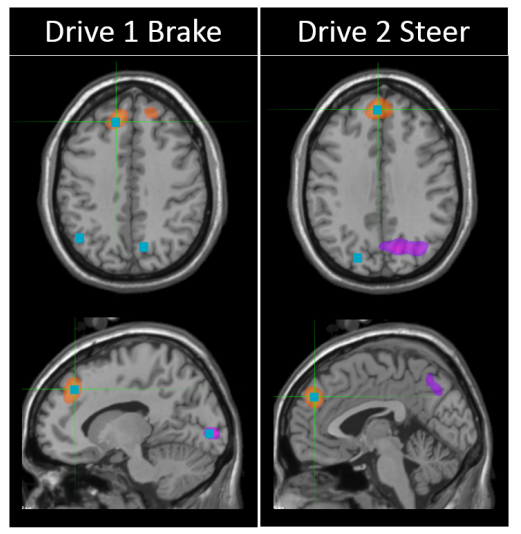

Figure 3. Differential image of increased power in superior frontal gyrus during driving.

Experiment 2. Examining grand averages of beta-band rhythm reflective of motor and somatosensory function (comparing rest and active driving), we saw similar beta-band ERD in hand areas of the motor cortex during steering on Drive 2 and Drive 3 (as above). In addition, the accelerate and brake responses across Drives 1 and 3 also had almost identical responses in the precentral gyrus of the motor cortex between $1-30 \mathrm{~Hz}$. However, we saw additional activity in theta-band $(4-7 \mathrm{~Hz})$, where power increased in frontal areas. See Figure 3.

\section{DISCUSSION}

This study successfully demonstrated a paradigm using driving simulator software and hardware in the metal/magnetically-sensitive MEG setting, without significant artifact. Our results were in line with our hypotheses regarding expected motor oscillations with increased motor control of the wheel and pedals. Furthermore, we were able to isolate frontal lobe neural activity beyond the motor cortex that is associated with cognitive processing (Cavanagh and Frank, 2014; Cavanagh and Shackman, 2015; Getzman et al., 2018).

Building on these results, further investigation is needed to identify consistency in these patterns of neural activity in a larger sample. Future studies may reveal additional frontal neural responses related to driving behaviors that may be modulated by task difficulty or load. With this paradigm, the level of complexity of the driving scene can be systematically increased by adding isolated elements (such as traffic, pedestrians, hazards and distractions) to test the limits of the neurocognitive mechanisms underlying driver behavior, both with and without advanced warning in-vehicle technology. Thus, this paradigm can be used to understand the neurocognitive 
basis of driver error, create new metrics for cognitive workload/distraction, and validate invehicle interventions that target attention.

In summary, this work has demonstrated, in a systematic way, the utility of an ecologicallyrelevant paradigm of combined MEG recording during simulated driving tasks of graded complexity. This methodological approach will allow us to not only examine whole cortex neural activity underlying driving behaviors and errors, but also quantitative biological markers sensitive to and predictive of the degree of capacity load and error during driving (by manipulating the complexity of the simulated driving scene). As such, this work has the potential to provide a theoretical foundation for understanding the neural mechanisms associated with driving performance. Furthermore, this paradigm can be used to examine the neural responses to in-vehicle technology and settings, thereby translating research between neuroscience, human factors, engineering and ergonomic, and in different populations at greater risk for driver error and crashes due to neurocognitive deficits or impairment, who may benefit the most from countermeasures.

\section{REFERENCES}

Singh, S. (2015, February). Critical reasons for crashes investigated in the National Motor Vehicle Crash Causation Survey. (Traffic Safety Facts Crash Stats. Report No. DOT HS 812 115). Washington, DC: National Highway Traffic Safety Administration.

Cavanagh, J. F., \& Frank, M. J. (2014). Frontal theta as a mechanism for cognitive control. Trends Cogn. Sci. 18, 414-421. doi: 10.1016/j.tics.2014.04.012

Cavanagh, J. F., \& Shackman, A. J. (2015). Frontal midline theta reflects anxiety and cognitive control: meta-analytic evidence. J. Physiol. Paris 109, 3-15.

Calhoun, V. D., Pekar, J. J., McGinty, V. B., Adali, T., Watson, T. D., \& Pearlson, G. D. (2002) Different activation dynamics in multiple neural systems during simulated driving. Hum Brain Mapp., 16, 158-67. doi: 10.1002/hbm.10032

Gaetz, W., Macdonald, M., Cheyne, D., \& Snead, O. C. (2010). Neuromagnetic imaging of movement-related cortical oscillations in children and adults: age predicts post-movement beta rebound. NeuroImage, 51:792-807.

Gaetz, W., Liu, C., Zhu, H., Bloy, L., \& Roberts, T. P. L. (2013). Evidence for a motor gammaband network governing response interference. NeuroImage, 74.

Lees, M. N., Cosman, J. D., Lee, J. D., Fricke, N., \& Rizzo, M. (2010). Translating cognitive neuroscience to the driver's operational environment: a neuroergonomic approach. The American journal of psychology, 123(4), 391-411.

Lerner, N., Singer, J., Huey, R., Brown, T., Marshall, D., Chrysler, S., ... \& Chiang, D. P. (2015). Driver-vehicle interfaces for advanced crash warning systems: Research on evaluation 
methods and warning signals. (Report No. DOT HS 812 208). Washington, DC: National Highway Traffic Safety Administration.

Mosquet, X., Andersen, M., \& Arora, A. A (2016). Roadmap to Safer Driving Through Advanced Driver Assistance Systems. Auto Tech Review, 5(7), pp. 20-25

Liu, T., Pelowski, M., Pang, C., Zhou, Y., \& Cai, J. (2016) Near-infrared spectroscopy as a tool for driving research. Ergonomics 59(3), 368-379

Papadelis, C., et al., (2016). Inferior frontal gyrus links visual and motor cortices during a visuomotor precision grip force task. Brain Res, 16(50), p. 252-266.

Robinson, S.E. \& Vrba, J. (1999), eds. Functional neuroimaging by synthetic aperture magnetometry. Biomag 2000: Proc. of the 12th Int. Conf. Biomag, ed. J. Nenonen, R.J. Ilmoniemi, and T. Katila. 1999, Helsinki University of Technology: Espoo, 1999. 302-305

Sakihara, K., Hirata, M., Ebe, K., Kimura, K., Yi Ryu, S., Kono, Y., Muto, N., Yoshioka, M., Yoshimine, T., ... \& Yorifuji, S. (2014). Cerebral oscillatory activity during simulated driving using MEG. Frontiers in human neuroscience, 8, 975. doi:10.3389/fnhum.2014.00975

Schweizer, T. A., Kan, K., Hung, Y., Tam, F., Naglie, G., \& Graham, S. J. (2013). Brain activity during driving with distraction: an immersive fMRI study. Frontiers in Human Neuroscience, 7 , 53. doi:10.3389/fnhum.2013.00053

Tallon-Baudry, C., (2012). On the neural mechanisms subserving consciousness and attention. Front Psychol, 2: p. 397.

Vrba, J. \& S. Robinson, (2001). Signal processing in magnetoencephalography. Methods (San Diego, Calif.), 25(2), p. 249-271.

Young, K. \& Regan, M. (2007). Driver distraction: A review of the literature. In: I.J. Faulks, M. Regan, M. Stevenson, J. Brown, A. Porter \& J.D. Irwin (Eds.). Distracted driving. Sydney, NSW: Australasian College of Road Safety. Pages 379-405.

Zhang, H., Chavarriaga, R., Khaliliardali, Z., Gheorghe, L., Iturrate, I., and Millán, J. d. R. (2015). EEG-based decoding of error-related brain activity in a real-world driving task. $J$. Neural. Eng. 12, 066028. doi: 10.1088/1741-2560/12/6/066028 